\title{
Microstructural Characterization of Pure Titanium Treated by Laser Surface Treatment Under Different Processing Parameters
}

\author{
Can Huang ${ }^{1,2} \cdot$ Jian $\mathrm{Tu}^{1,2} \cdot$ Yu-Ren $\mathrm{Wen}^{3} \cdot \mathrm{Zhi} \mathrm{Hu}^{4} \cdot$ Zhi-Ming Zhou ${ }^{1,2} \cdot$ An-Ping Dong ${ }^{4} \cdot$ Guo-Liang Zhu ${ }^{4}$
}

Received: 15 March 2017/Revised: 20 April 2017/Published online: 12 June 2017

(C) The Chinese Society for Metals and Springer-Verlag GmbH Germany 2017

\begin{abstract}
Advanced characterization techniques are utilized to investigate the effect of laser surface treatment on microstructural evolution of pure titanium (Ti). The results show that there are three distinctly different types of microstructure from surface to substrate in Ti samples, including phase transformation and solidification microstructure in zone I (melting zone); insufficient recrystallization grains with residual $\alpha$ martensitic plates in zone II (heat-affected zone, HAZ); fully recrystallization microstructure in zone III (base metal, BM). The hardness evolution profiles under different laser treatment parameters are similar. The highest hardness in MZ is ascribed to $\alpha$ plate, while the lowest hardness value in HAZ is due to the insufficiently recrystallized grains. The metallurgical process on the laser-modified Ti samples is systematically discussed in this work.
\end{abstract}

KEY WORDS: Laser surface treatment; Microstructure characterization; Titanium

\section{Introduction}

Commercially pure titanium (Ti) has the high strength-toweight ratio, low-cost manufacturing, excellent corrosion resistance and superior fracture toughness. However, poor

Available online at http://link.springer.com/journal/40195

Jian Tu

tujian@cqut.edu.cn

$\triangle$ An-Ping Dong

apdong@sjtu.edu.cn

1 School of Materials Science and Engineering, Chongqing University of Technology, Chongqing 400054, China

2 Chongqing Municipal Key Laboratory of Institutions of Higher Education for Mould Technology, Chongqing University of Technology, Chongqing 400054, China

3 Beijing National Laboratory for Condensed Matter Physics, Institute of Physics, Chinese Academy of Sciences, Beijing 100190, China

4 Shanghai Key Lab of Advanced High-temperature Materials and Precision Forming, Shanghai Jiao Tong University, Shanghai 200240, China surface fatigue resistance, low hardness and wear resistance of Ti would lead to fatigue failure [1, 2]. In addition, Ti alloys are used in bio-implant application due to its relatively low Young's modulus. However, the problems in long-term use of $\mathrm{Ti}$ alloys as medical implant are the bioinertness and poor adhesion [3, 4]. It is worth noting that surface engineering aims to tailor the microstructure and composition of the near-surface region, improving mechanical properties without affecting the substructure of bulk material $[5,6]$. Thus, surface engineering can offer significant potential for improving near-surface properties of $\mathrm{Ti}$ and its alloys.

Laser surface treatment (one of surface engineering technologies) is a convenient and economical processing method [7, 8]. Laser surface treatment has a wide application in industry due to its precision of operation and production of a modified region. In addition, laser surface treatment can bring non-equilibrium systems and generation of rapid solidification microstructure in the nearsurface region, offering significant potential for near-surface properties $[9,10]$. Thus, many achievements on Febased alloys [11-15], Mg-based alloys [16, 17] and Tibased alloys [18-23] have been reached. For example, 
laser surface treatment in argon atmosphere significantly refined the microstructure without carbides and martensite in tool steels, and the micro-hardness increases about twice as compared to the as-received sample [14]. In addition, the corrosion resistance of laser-treated surface in AZ91 Mg alloy was significantly enhanced due to the refined continuous network of $\mathrm{Mg}_{17} \mathrm{Al}_{12}$ phases [16]. Furthermore, the improved pitting corrosion resistance in pure $\mathrm{Ti}$ is obtained due to the microstructural changes caused by rapid solidification during laser surface treatment [24]. To the best of the authors' knowledge, microstructural characteristics by electron backscatter diffraction (EBSD) technology in laser-modified Ti are still limited. For better understanding the effect of laser surface treatment on the macro-properties and microstructure of $\mathrm{Ti}$ and its alloys, it is critical to evaluate the key influential parameters, especially, laser power output. With these in mind, the microstructural evolution in response to laser surface treatment at different powers is investigated by EBSD, aiming at achieving a uniform and sound-modified region.

\section{Experimental}

The as-received $\mathrm{Ti}$ sheets (99.9\%) with $2 \mathrm{~mm}$ thick are fully recrystallization. Figure 1a is an inverse pole figure (IPF) map, showing that many grains have their $c$-axes close to the normal direction (ND) of Ti sample. $\{0001\}$ pole figure in Fig. 1b presents a typical bimodal basal texture with each density maximum inclined around $20^{\circ}$ from the ND toward the transverse direction (TD). In addition, the $\{10 \overline{1} 0\}$ poles are aligned with the rolling direction (RD). The above microstructure features can be representative for a recrystallized $\mathrm{Ti}$ sample. There are
Table 1 Laser processing parameters for Ti samples

\begin{tabular}{lccc}
\hline Parameter & Specimen 1 & Specimen 2 & Specimen 3 \\
\hline Single pulse peak (kw) & 1 & 1 & 2 \\
Single pulse width (ms) & 4 & 8 & 4 \\
Laser energy input (J) & 4 & 8 & 8 \\
Pulse frequency (HZ) & 20 & 20 & 20 \\
Laser power output (W) & 80 & 160 & 160 \\
\hline
\end{tabular}

differences in the standard pole figure due to the different ratios of $c / a$ with respect to the hexagonal materials [25]. Thus, it is suitable for Ti material with basal pole spreading since its $c / a$ ratios (1.587) are smaller than the ideal ration (1.624) [25].

The samples with dimensions of 5,8 and $2 \mathrm{~mm}$ along $\mathrm{RD}, \mathrm{TD}$ and ND were cut from the received Ti sheets. Prior to laser treatment, the sample surfaces were polished with $\mathrm{SiC}$ papers with different grades of roughness, followed by cleaning in deionized water. Ultrasound in ethanol was used to degrease the sample surface, and the single track of laser surface treatment was conducted. The RD-TD surfaces were processed along TD by a pulsed $600 \mathrm{~W} \mathrm{Nd}$ : YAG laser device. The laser processing parameters are shown in Table 1 . The other constant parameters are laser beam scan speed of $15 \mathrm{~mm} / \mathrm{s}$, laser beam spot diameter of $1 \mathrm{~mm}$ and defocusing amount of $+2 \mathrm{~mm}$. The laser powers are set with varying the pulse width (specimen 1 and specimen 3) or pulse peak (specimen 1 and specimen 2).

Microstructure of RD-ND planes were characterized by emission gun scanning electron microscope (SEM). Prior to SEM examinations, all specimens were mechanically ground using $\mathrm{SiC}$ paper (3000\# at the final step), and then electro-polishing was conducted at $17 \mathrm{~V} / 0.6 \mathrm{~A}$ at $-20{ }^{\circ} \mathrm{C}$ for $70 \mathrm{~s}$ in a solution, which consisted of $90 \mathrm{ml}$

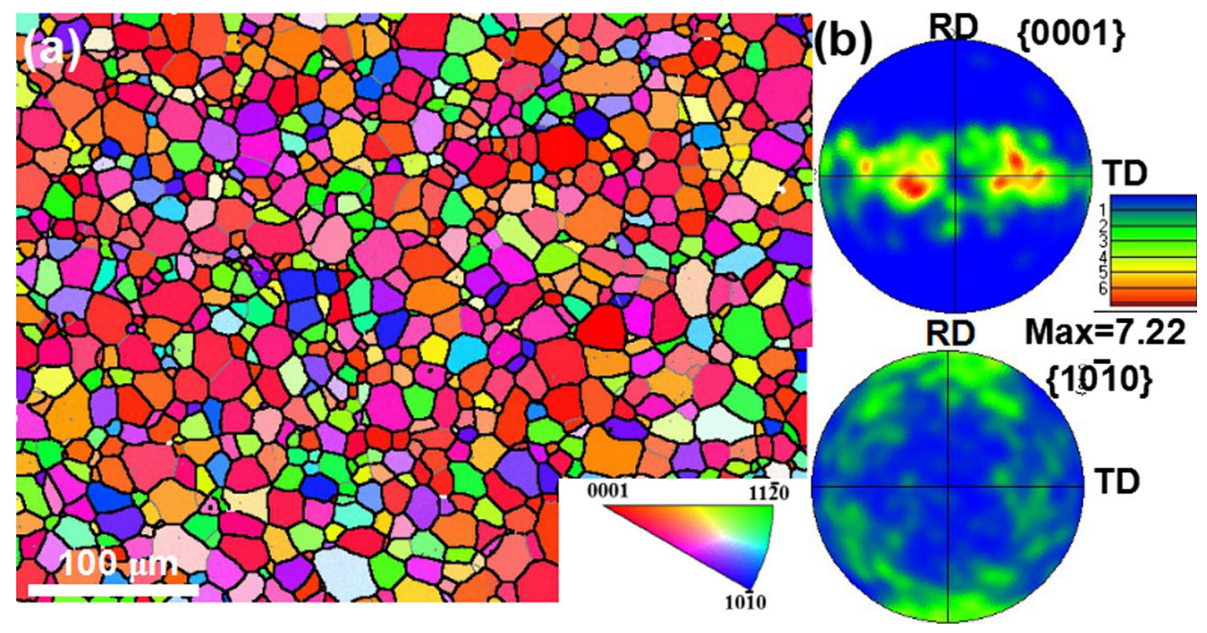

Fig. 1 Inverse pole figure a and $\{0001\}$ and $\{10 \overline{1} 0\}$ pole figures $\mathbf{b}$ of recrystallized Ti sample 
formaldehyde, $10 \mathrm{ml}$ perchloric acid. The specimens were characterized by electron channeling contrast (ECC) imaging techniques in a Zeiss Sigma HD field-emission gun SEM. In addition, step sizes used for the EBSD scanning varied from 0.25 to $1.2 \mu \mathrm{m}$, depending on their grain sizes. The EBSD patterns were processed by using the Channel 5 software from HKL technology to determine the local misorientation. Micro-hardness distributions were examined using a HVS-1000 micro-hardness tester (Vickers indenter). Each indentation is done under a load of HV0.5 with holding time $10 \mathrm{~s}$. The values at ten various locations with the same depth were measured and then averaged, obtaining the reliable hardness data.

\section{Results and Discussion}

Figure 2 shows the surface morphologies of $\mathrm{Ti}$ samples processed by different laser powers. The crescent-shape solidification ripples arise at the top of solidified molten pools, and these molten pools exhibit "dropout" morphology. Obviously, the molten zone has the brighter contrast than the unaffected substrate. Compared with specimen 1 , the sizes of bead width and the penetration depth are larger in specimen 2 and specimen 3. Compared with specimen 1 and specimen 2, a more rough surface finish in molten pool with the severe vibration is observed in specimen 3 .

\subsection{Cross-Sectional Observations by ECC}

The cross-sectional views (RD-ND plane) are exhibited to comprehensively understand the microstructural evolution of the laser-modified regions. Figure $3 \mathrm{a}$ shows the crosssectional view of molten pool for specimen 1 (1 $\mathrm{kw} \times 4 \mathrm{~ms}$ ) by ECC technology at low magnification. The laser heat generation at the middle portion of modified zone is higher than that along the circumference [9]. Thus, the heat generation at the middle portion of modified region is higher than those at the edges, leading to a bowl-like cross- sectional morphology, as shown in Fig. 3a. Three distinctive zones are outlined by two dashed lines, including melting zone (MZ, zone I), heat-affected zone (HAZ, zone II) and base metal (BM, zone III). Some $\alpha$ martensitic plate appears inside prior austenite grains, as shown in zone I in Fig. $3 b$ at a higher magnification. The coarsened grains with irregular shape and inhomogeneous size are revealed in zone II (Fig. 3a). The prior equiaxed grains are completely maintained in zone III (Fig. 3a), which correspond to the unaffected substrate. Compared with specimen 1 , the similarity of general microstructural characteristics for specimen $2(1 \mathrm{kw} \times 8 \mathrm{~ms})$ is shown in Fig. 3c. Moreover, Fig. $3 d$ shows the denser $\alpha$ martensitic plate in either parallel or inter-sectional forms. Interestingly, Fig. 3e shows that the rod-shaped grains are formed in zone I for specimen $3(2 \mathrm{kw} \times 4 \mathrm{~ms})$, which is considered as the elongated columnar structure (solidification microstructure) [26]. The columnar grains for zone I in Fig. 3e are magnified in Fig. 3f, showing the coarsened grains with a certain direction.

When Ti sample is impacted by laser, the outermost surface layer begins to melt [27], and the morphological characteristics of metallic surface are shown in Fig. 2. The laser pulse duration is very short, so the laser-induced heat is released through the cold substrate, leading to a selfquenching effect in zone I. Thus, the rapid $\beta \rightarrow \alpha$ phase transformation will occur, resulting in $\alpha$ martensitic plate in zone I (Fig. 3a). It is also worth noting that the selfquenching effect also initiates in zone II, but the laserinduced heat effect is weaker in zone II. Therefore, much more less $\alpha$ martensitic plate can be produced in zone II (Fig. 3c). Subsequently, cooling process in zone I makes the excessive heat to dissipate through zone II into the cold substrate (zone III). Thus, the recrystallization process would be operated in zone II. Once enough heat is captured, some grains recrystallized in zone II. In addition, the coarsening recrystallization grains in zone II are also revealed (Fig. 3a, c).No microstructure changes happen in zone III due to that there is no laser-induced heat effect in zone III (Fig. 3a, c, e).
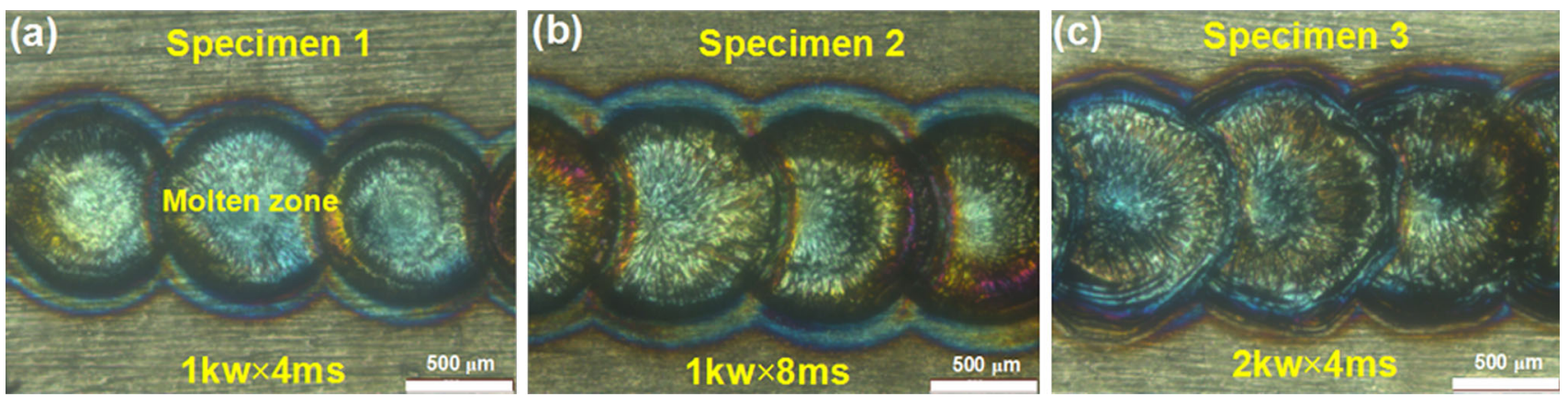

Fig. 2 Optical microscope images of laser-treated surfaces for specimen 1 a, specimen 2 b specimen 3 c 

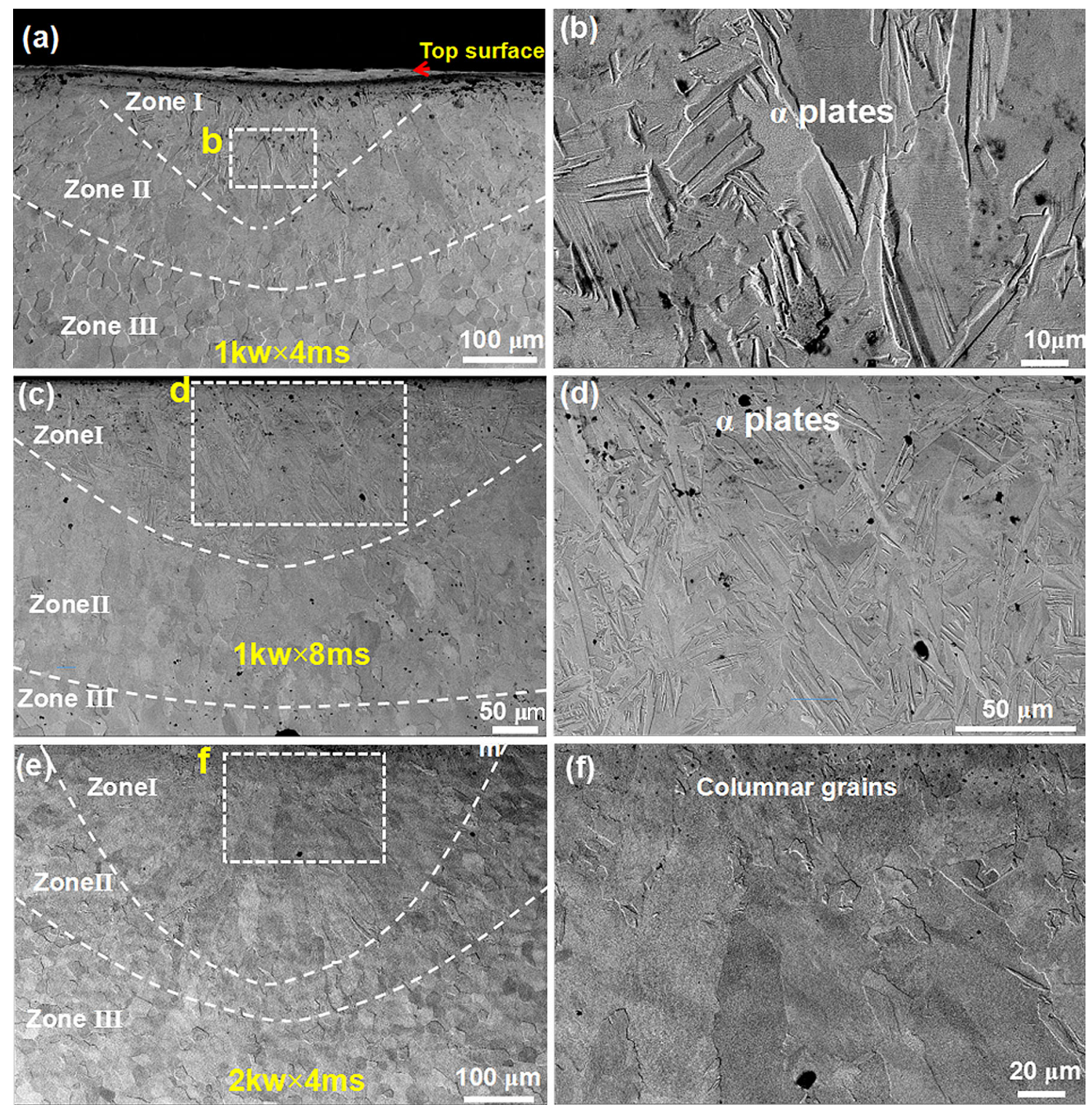

Fig. 3 Electron channeling contrast showing microstructural characteristics of cross-sectional views of specimen 1 a, b, specimen 2 c, d and specimen $3 \mathbf{e}, \mathbf{f}$ with three distinctly different zones including zone I (melting zone), zone II (heat-affected zone) and zone III (base metal) a, c, e $\alpha$ martensitic plates in zone I $\mathbf{b}, \mathbf{d}$ columnar grains in zone I $\mathbf{f}$

\subsection{Cross-Sectional Observations by EBSD}

The bowl-like profiles are exhibited in the all Euler angle map superposed by a band contrast map (Fig. $4 \mathrm{a}-\mathrm{c}$ ). The transition lines between the modified region (zone I and zone II) and the unaffected region (zone III) are marked. For specimen $1(1 \mathrm{kw} \times 4 \mathrm{~ms})$ and specimen $2(1 \mathrm{kw} \times 8 \mathrm{~ms})$ with the same peak power $(1 \mathrm{kw})$, the laser impact time increases as the pulse duration increases, leading to the higher heat input in specimen 2 . Thus, the stronger selfquenching effect is in specimen 2, revealing the denser $\alpha$ martensitic plates in MZ (Fig. 4a, b). For specimen 1 (1 $\mathrm{kw} \times 4 \mathrm{~ms})$ and specimen $3(2 \mathrm{kw} \times 4 \mathrm{~ms})$ with the same pulse duration, the higher heat input is produced in specimen 3 as the peak power increase, leading to the longer cooling time under the higher heat input (specimen 3 ). Thus, the typical solidification microstructure is exhibited in specimen 3 (Fig. 4c). Interestingly, for specimen $2(1 \mathrm{kw} \times 8 \mathrm{~ms})$ and specimen $3(2 \mathrm{kw} \times 4 \mathrm{~ms})$ under the same heat input $(8 \mathrm{~J})$, completely different microstructural characteristics in zone I are revealed in MZ: Specimen 3 shows the solidification microstructure (columnar grains), while specimen shows the phase transformation microstructure ( $\alpha$ martensitic plates) in specimen 2. Much more microstructural details for specimen 1, specimen 2 and specimen 3 are shown in Figs. 5, 6 and 7, respectively. 

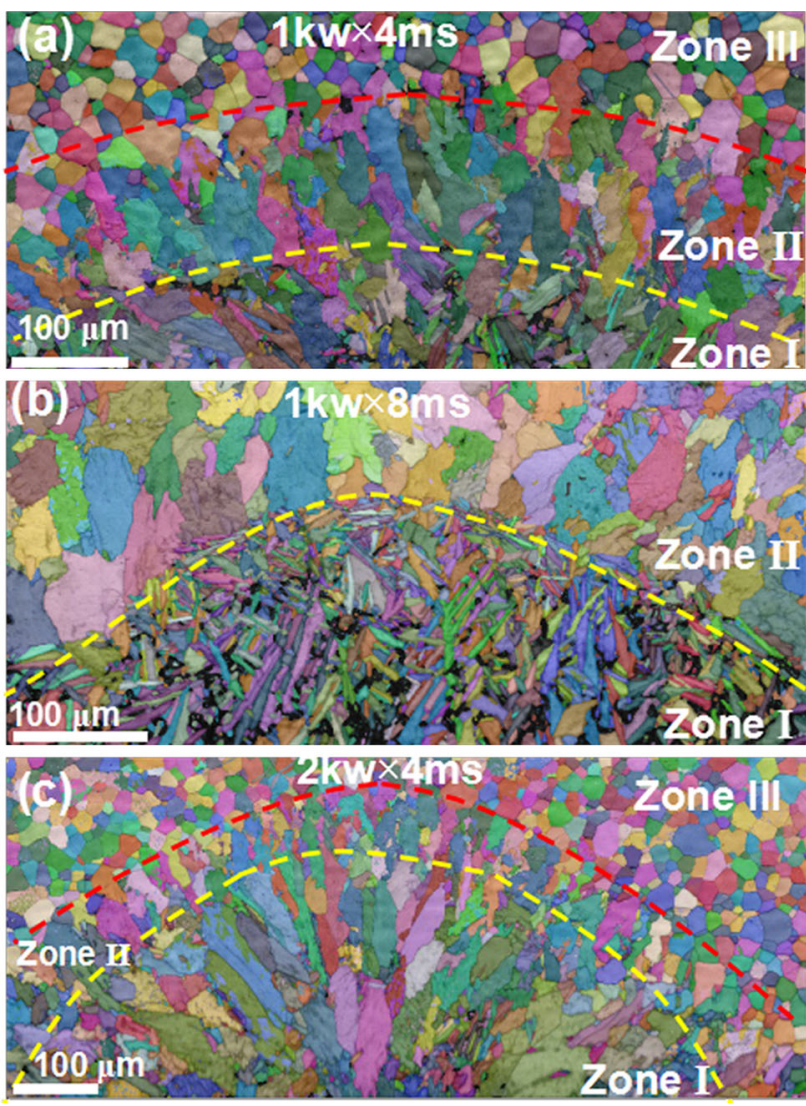

Fig. 4 All Euler angle map superposed by a band contrast map showing bowl-like profiles in laser-modified Ti samples for specimen 1 a, specimen 2 b specimen 3 c

Figure 5 shows the EBSD map scanning at a step size of $0.6 \mu \mathrm{m}$ for specimen 1 . Three distinct zones can be distinguished in Fig. 5a: Some $\alpha$ martensitic plates appear inside austenite grains in zone I; the coarsened grains with irregularity in shape exist in zone II; and the fully recrystallization microstructure occurs in zone III. Burgers orientation relationship (OR) predicts five specific misorientations $\quad\left(10.5^{\circ}<0001>, \quad 60^{\circ}<11 \overline{2} 0>\right.$, $\sim 60.8^{\circ}<\overline{4} \overline{3} 71>, \sim 63.3^{\circ}<\overline{4} 221>, \sim 90^{\circ}<3 \overline{7} 40>$ ) during $\beta \rightarrow \alpha$ transformation in $\mathrm{Ti}$ [28]. The special boundaries (SBs) inside prior austenite grains belonging to the five Burgers misorientations can be detected in GB map (Fig. 5b). These SBs are marked by different colored lines, in which the frequency of $10.5^{\circ}<0001>$ boundary is much lower than other SBs. In addition, high-angle boundaries (HABs with misorientation angle higher than $14^{\circ}$ ) and low-angle boundaries (LABs with misorientation angle between $2^{\circ}$ and $7^{\circ}$ ) are represented by black and gray lines. The thermal stress induced by laser can produce LABs.

Three different zones for specimen $2(1 \mathrm{kw} \times 8 \mathrm{~ms})$ are shown in Fig. 6a (zone I and zone II) and Fig. 6b (zone II and zone III). For zone I, GB map in Fig. 6c shows the overwhelming majority of the detected grain boundary misorientations belong to two Burgers misorientations $\left(60^{\circ}<11 \overline{2} 0>, 63.3^{\circ}<\overline{4} 221>\right)$. Indeed, other Burgers misorientations $\quad\left(10.5^{\circ}<0001>, \quad 60.8^{\circ}<\overline{4} \overline{3} 71>\right.$, $90^{\circ}<3 \overline{7} 40>$ ) are existed; but their fraction is relatively low. Thus, the variant selection phenomenon $\left(60^{\circ}<11 \overline{2} 0>\right.$ and $\left.63.3^{\circ}<\overline{4} 221>\right)$ has occurred in zone I for specimen 2 . The variant selection can induce the "texture memory" phenomenon [29-31], affecting the mechanical performance of $\mathrm{Ti}$ materials after $\beta \rightarrow \alpha$ transformation. The corresponding rotation axis distributions around the Burgers misorientation of $8^{\circ}-13^{\circ}, 57^{\circ}-66^{\circ}$ and $87^{\circ}-90^{\circ}$ are confirmed in Fig. 6f. For the insufficiently recrystallized structures in zone II in Fig. 6d, they are believed to evolve from the previous $\alpha$ martensitic plate substructure, which are formed by the transferring heat form zone I to zone II. As a result, the insufficient recrystallized grains with the residual $\alpha$ martensitic plates are formed in zone II. The existence of a number of LABs in Fig. 6d further confirms the insufficient recrystallization

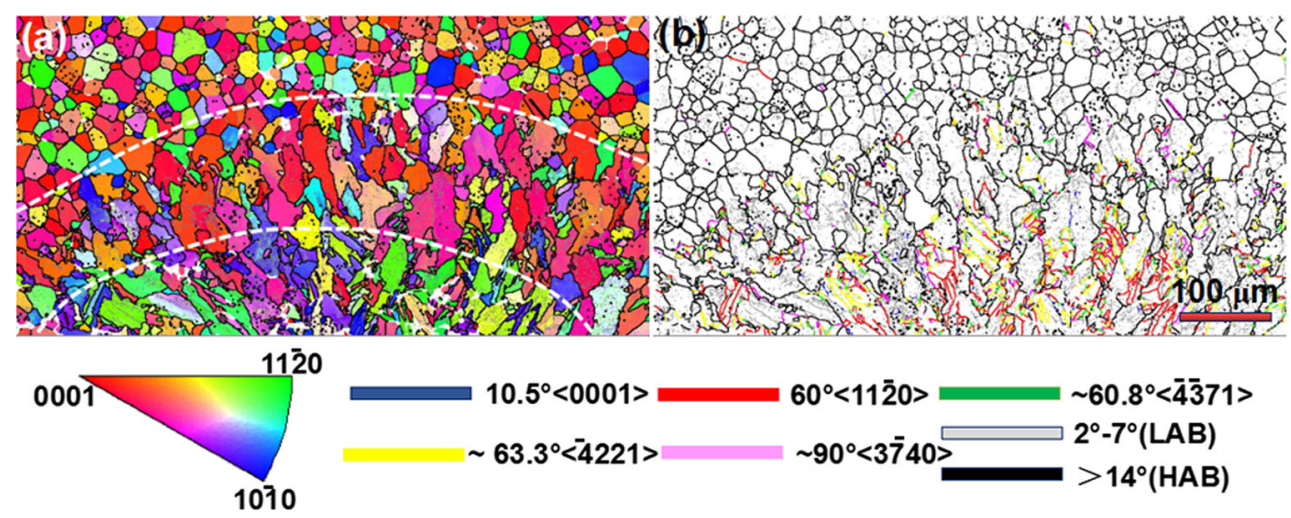

Fig. 5 EBSD maps of specimen 1 showing three zone, including melting zone (MZ), heat-affected zone (HAZ) and base metal (BM) which are separated by two broken lines a and grain boundary map showing special boundaries with Burgers misorientation (marked by different colored lines) $\mathbf{b}$ 


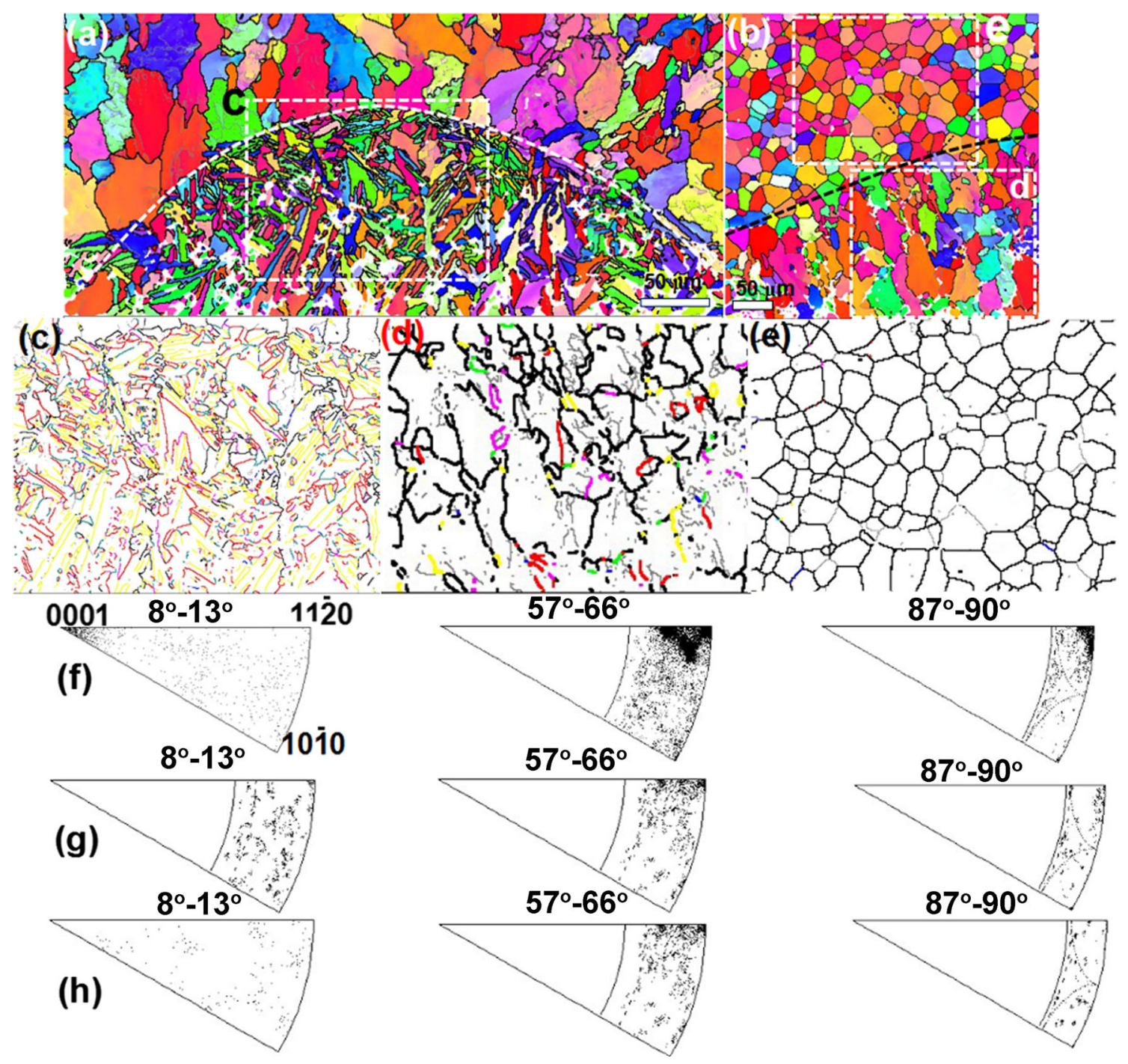

Fig. 6 EBSD maps showing microstructure characteristics for specimen 2: a zones I and II; b zones II and III; c GB map for zone I; d GB map for zone II; e GB map for zone III; $\mathbf{f}-\mathbf{g}$ rotation axis distributions around Burgers misorientation of $8^{\circ}-13^{\circ}, 57^{\circ}-66^{\circ}$ and $87^{\circ}-90^{\circ}$ for zone I, zone II and zone III, respectively

in zone II. These LABs with the misorientation angles lower than $8^{\circ}$ (Fig. $6 \mathrm{~d}$ ) could be resulted from the recovery of quenching-induced dislocation [28]. Figure $6 \mathrm{~g}$ displays the rotation axis distributions around the Burgers misorientation of $8^{\circ}-13^{\circ}, 57^{\circ}-66^{\circ}$ and $87^{\circ}-90^{\circ}$ for zone II. Figure $6 \mathrm{e}$ and $\mathrm{h}$ displays the GB map for zone III and their rotation axis distributions around the Burgers misorientation of $8^{\circ}-13^{\circ}, 57^{\circ}-66^{\circ}$ and $87^{\circ}-90^{\circ}$, suggesting the fully recrystallized microstructure without any changes.

Figure 7 shows the microstructure characteristics for specimen $3(2 \mathrm{kw} \times 4 \mathrm{~ms})$. Specifically, IPF map in Fig. $7 \mathrm{a}$ shows the typical solidification microstructure (columnar grains) with a length of millimeter in zone I. Three distinct zones in one area can be easily distinguished in Fig. 7b, clearly showing their respective microstructural characteristics. GB maps in Fig. 7d show that the possible misorientations among $\alpha$ plate are detected in columnar grains, suggesting that $\beta \rightarrow \alpha$ phase transformation also occurs after solidification process in zone I in specimen 3 . As compared to the zone II for specimens 1 and 2, the uncompleted recrystallized GB characteristics are also revealed in zone II for specimen 3 (Fig. 7c). Interestingly, the solidification microstructure is only revealed in specimen 3 due to the longer cooling time. Its solidification process can be described as follows: During the molten metal solidification process, the grains tend to grow in the direction perpendicular to pool boundary because this is the direction of the maximum temperature gradient and hence maximum heat extraction $[7,9,10]$. However, columnar grains tend to grow in the easy-growth $<10 \overline{1} 0>$ direction [32]. Therefore, solidification grains with their most favorable direction essentially perpendicular to the pool 


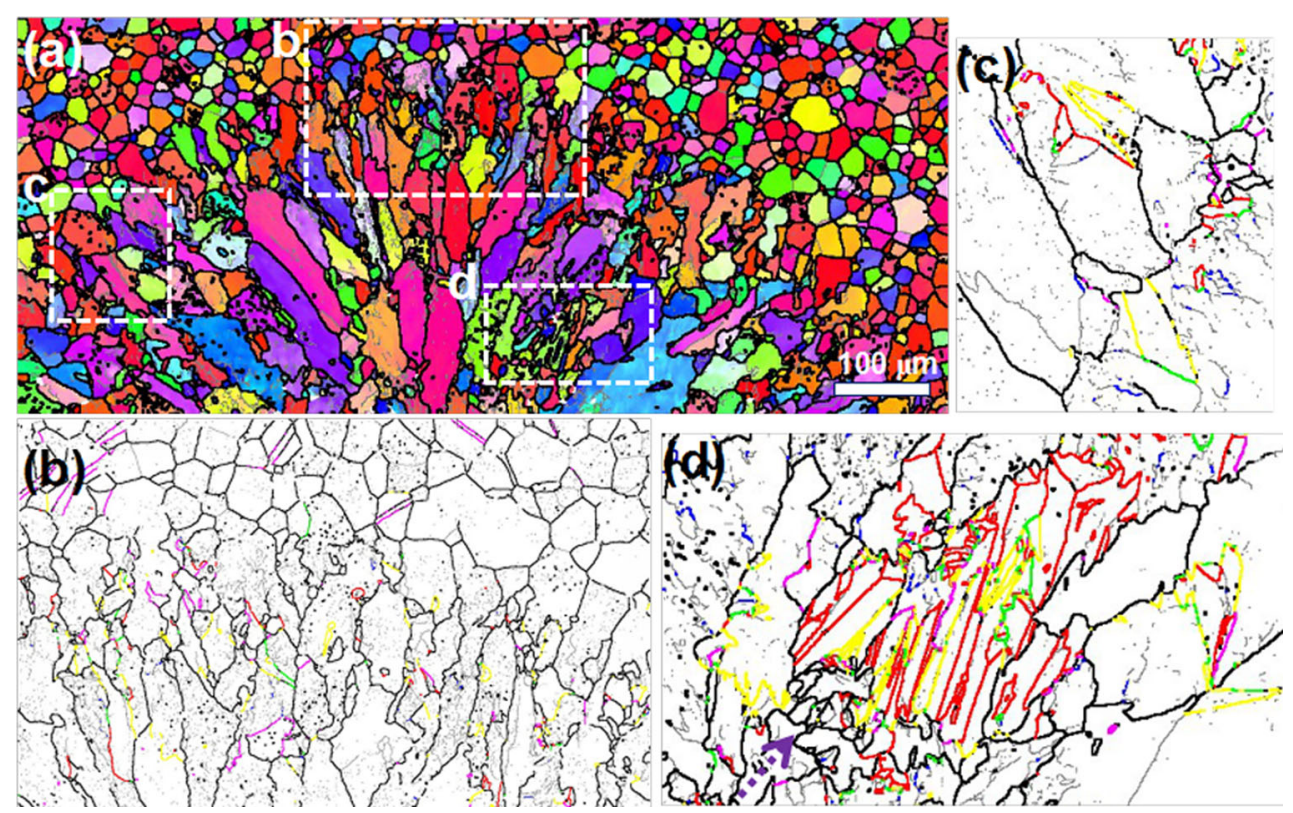

Fig. 7 EBSD maps showing microstructure characteristics for specimen 3: a three different zones; b GB map for zone I; c GB map for zone II; d GB map for zone III

boundary will grow more easily and crow out those less favorably oriented grains. This competitive growth mechanism dominates the growth direction of columnar grains. Thus, the parent $\gamma$ grains are elongated along the thermal gradient direction, resulting in the rod-shaped grains in MZ. In addition, most of these columnar grains, which are separated from one another by high-angle boundaries, are always aligned as anisotropy (IPF in Fig. 7a).

\subsection{Hardness}

In addition to the microstructural characteristics as described above, the hardness of different zones for specimens 1,2 and 3 is shown in Fig. 8c, respectively. An average hardness value of $167.2 \mathrm{HV}$ is located in zone III (BM). Although the different modified depth can be obtained under different laser processing parameters, the similarity of general hardness evolution profiles can be seen Fig. 8. In zone I, the micro-hardness reaches the highest value at a site near the top surface. For specimen 2, zone $\mathrm{I}$ is found to be generally much harder than BM and the highest hardness value is 459.9 HV. Furthermore, specimen 2 has the highest hardness value in zone I than the two other specimens. These are ascribed to the denser $\alpha$ plates in specimen 2, leading to the significant grain refinement.

The lowest hardness value is always located in zone II, which is determined to be generally softer than the substrate. This may be ascribed to the very heterogeneous grain structures in zone II, where the coarsening grains are found to exist extensively. These coarsening grains in zone II would contribute less to the hardness than that in the substrate. Although there are some LABs and $\alpha$ boundary inside these un-completed grains, the overall impediment (a)

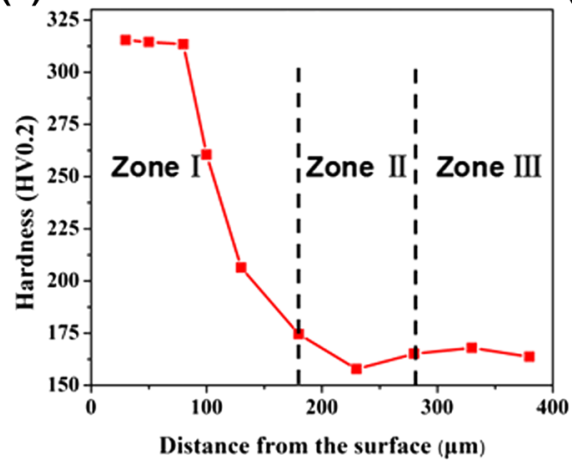

(b)

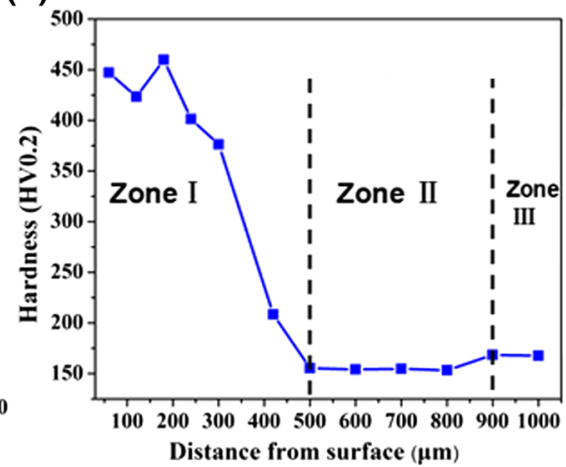

(c)

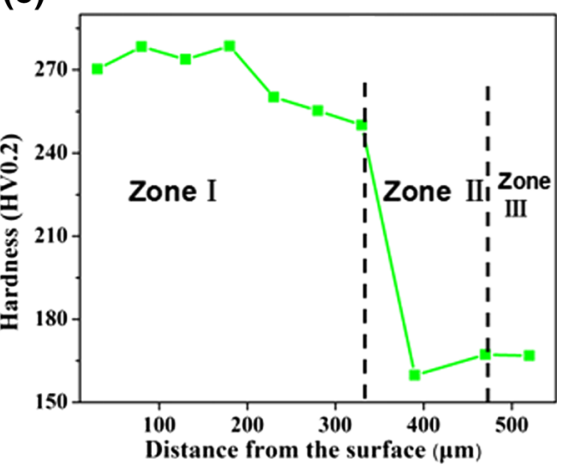

Fig. 8 Micro-hardness variations along depth from surface including MZ, HAZ and BM for specimen 1 a, specimen 2 b specimen 3 c 
to dislocation motion provided by them remains relatively weak. Thus, the hardness in zone II is even lower than the hardness in BM (Fig. 8).

\section{Conclusions}

The systematical EBSD characterization confirms that laser surface treatment for Ti samples under different processing parameters can markedly affect their microstructural characteristics. Some key conclusions have been drawn as follows:

1. After the laser surface treatment, three distinctly different microstructural features are exhibited: zone I (melting zone) for phase transformation microstructure in prior austenite grain (specimen 1 and specimen 2) or solidification microstructure in elongated columnar grains (specimen 3); zone II (heat-affected zone) for insufficient recrystallization grains with residual $\alpha$ martensitic plates; and zone III (base metal) for fully recrystallization grains.

2. The similarity of general hardness evolution profiles in different laser treatment parameters. In zone I, the micro-hardness is highest due to the denser $\alpha$ plates. In zone II, the micro-hardness always has the lowest value, which is ascribed to the insufficiently recrystallized grains.

Acknowledgements This work was supported by the National Natural Science Foundation of China (Nos. 51401039 and 51501026), the Natural Science Foundation of Chongqing (No. CSTC2014jcyjA50017), the Scientific and Technological Research Program of Chongqing Municipal Education Commission (Nos. KJ1500923 and KJ1709204).

\section{References}

[1] C. Leyens, M. Peters, Titanium and Titanium Alloys (WileyVCH, Köln, 2003)

[2] G. Lütjering, J.C. Williams, Titanium (Springer, Heidelberg, 2003), p. 149

[3] M. Geetha, A.K. Singh, R. Asokamani, A.K. Gogia, Prog. Mater. Sci. 54, 397 (2009)

[4] E. Mohseni, E. Zalnezhad, A.R. Bushroa, Int. J. Adhes. Adhes. 48, 238 (2014)

[5] N. Huang, Y.X. Leng, P.D. Ding, Surface Engineering of Light Alloys (Woodhead Publishing, Oxford, 2010), p. 568
[6] H. Dong, Surface Engineering of Light Alloys: Aluminium, Magnesium and Titanium Alloys (Woodhead Publishing Limited, Oxford, 2010)

[7] J. Kusinski, S. Kac, A. Kopia, A. Radziszewska, M. RozmusGórnikowska, B. Major, L. Major, J. Marczak, A. Lisiecki, Bull. Polish Acad. Sci. Tech. Sci. 60, 711 (2012)

[8] C.T. Kwok, Laser Surface Modification of Alloys for Corrosion and Erosion Resistance (Woodhead Publishing Limited, Oxford, 2012)

[9] E. Kannatey, Principles of Laser Materials Processing (Wiley, Hoboken, 2009)

[10] J.D. Majumdar, I. Manna, Sadhana 28, 495 (2003)

[11] A. Conde, I. García, J. De Damborenea Corros. Sci. 43, 817 (2001)

[12] Y. Yoon, J. Yim, E. Choi, J. Kim, K. Oh, Y. Joo, Mater. Lett. 185, 43 (2016)

[13] C. Ye, S. Suslov, B.J. Kim, E.A. Stach, G.J. Cheng, Acta Mater. 59, 1014 (2011)

[14] J.D. Majumdar, A. Nath, I. Manna, Surf. Coat. Technol. 204, 1326 (2010)

[15] A. de Pablos-Martín, C. Grosse, A. Cismak, T. Höche, Acta Metall. Sin. (Eng. Lett.) 29, 683 (2016)

[16] Y. Guan, W. Zhou, H. Zheng, J. Appl. Electrochem. 39, 1457 (2009)

[17] A. Singh, S.P. Harimkar, JOM 64, 716 (2012)

[18] F. Weng, C. Chen, H. Yu, Mater. Des. 58, 412 (2014)

[19] X. Nie, W. He, S. Zang, X. Wang, J. Zhao, Surf. Coat. Technol. 253, 68 (2014)

[20] Y.S. Tian, C.Z. Chen, S.T. Li, Q.H. Huo, Appl. Surf. Sci. 242, 177 (2005)

[21] Z. Ding, C. Zhang, L. Xie, L.C. Zhang, L. Wang, W. Lu, Metall. Mater. Trans. A 47, 5675 (2016)

[22] N. Dai, L.C. Zhang, J. Zhang, Q. Chen, M. Wu, Corros. Sci. 102, 484 (2016)

[23] Y. Chen, J. Zhang, N. Dai, P. Qin, H. Attar, L.C. Zhang, Electrochim. Acta 232, 89 (2017)

[24] Z. Sun, I. Annergren, D. Pan, T.A. Mai, Mater. Sci. Eng. A 345, $293(2003)$

[25] Y.N. Wang, J.C. Huang, Mater. Chem. Phys. 81, 11 (2003)

[26] W.J. Boettinger, D.K. Banerjee, in Physical Metallurgy, ed. by D.E.L. Hono, 5th edn (Elsevier, Oxford, 2014) pp. 639-850

[27] P.M.O. Marta Castillejo, L. Zhigilei, Lasers in Materials Science (Springer, Heidelberg, 2014)

[28] S.D. Banerjee, P. Mukhopadhyay, Phase Transformations: Examples from Titanium and Zirconium Alloys (Elsevier, Amsterdam, 2010)

[29] S. Xu, L.S. Toth, C. Schuman, J.S. Lecomte, M.R. Barnett, Acta Mater. 124, 59 (2017)

[30] G. Obasi, S. Birosca, J.Q. da Fonseca, M. Preuss, Acta Mater. 60, 1048 (2012)

[31] Z. Li, J. Li, Y. Zhu, X. Tian, H. Wang, J. Alloys Compd. 661, $126(2015)$

[32] S. Kou, Welding Metallurgy (Cambridge University Press, New York, 1987) 